\title{
Diacronie
}

Studi di Storia Contemporanea

$N^{\circ} 18,2 \mid 2014$

Le esposizioni: propaganda e costruzione identitaria

\section{Nota introduttiva n. 18 - giugno 2014}

\section{Francescomaria Evangelisti e Alessandro Pes}

\section{(2) OpenEdition}

\section{Journals}

\section{Edizione digitale}

URL: http://journals.openedition.org/diacronie/1156

DOI: 10.4000/diacronie.1156

ISSN: 2038-0925

\section{Editore}

Association culturelle Diacronie

Notizia bibliografica digitale

Francescomaria Evangelisti e Alessandro Pes, « Nota introduttiva n. 18 - giugno 2014 », Diacronie [Online], $\mathrm{N}^{\circ}$ 18, 2 | 2014, Messo online il 01 juin 2014, consultato il 22 septembre 2020. URL : http:// journals.openedition.org/diacronie/1156; DOI : https://doi.org/10.4000/diacronie.1156

Questo documento è stato generato automaticamente il 22 settembre 2020.

Creative Commons License 


\title{
Nota introduttiva n. 18 - giugno 2014
}

\author{
Francescomaria Evangelisti e Alessandro Pes
}

1 Pubblicare a pochi mesi dall'inaugurazione dell'Esposizione di Milano 2015 un numero dedicato all'analisi storica di questo fenomeno potrebbe sembrare un'operazione pianificata prevedendo gli scandali, i ritardi e la retorica che ne avrebbero accompagnato l'avvento nell'opinione pubblica italiana e internazionale. Questa

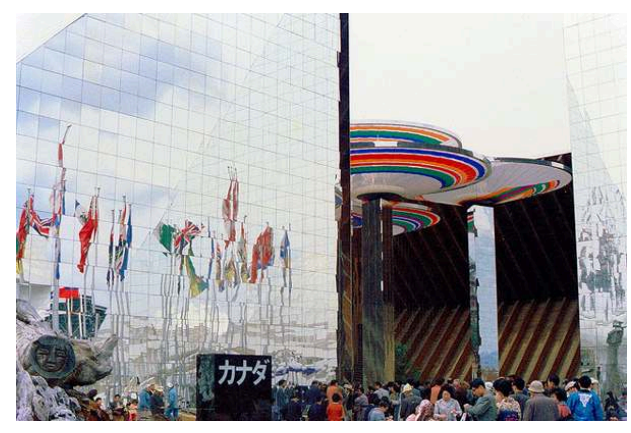
supposizione è certa perché soprattutto in un momento in cui essa sembra essersi svuotata del suo senso originario per convertirsi in una costosa vetrina che attira appetiti speculatori, è necessario analizzare qual è stata la sua influenza nella cultura e nella diplomazia degli ultimi tre secoli.

2 Le origini delle esposizioni rimandano all'Imperialismo ${ }^{1}$ e al capitalismo industriale e le configurano come un dispendioso ma efficace strumento propagandistico in mani borghesi e aristocratiche che ha il vantaggio di agire in un clima di divertimento ${ }^{2}$, Lotze la definiva infatti "l'unica vera festa moderna".

3 Nazioni ed imperi si contendevano la possibilità di erigere nelle loro capitali gli altari per celebrare quel che Hobsbwam ${ }^{3}$ definisce come un nuovo, allegorico e gigantesco rituale di auto-esaltazione del capitalismo e dell'industrializzazione ${ }^{4}$, propagata come uno strumento fondamentale per garantire Progresso e Pace.

4 L'idolo di questa celebrazione è il prodotto, risultato del unione tra capitale borghese e lavoro proletario, del genio nazionale o di quello artistico, inserito in un contesto che aggiunge, al suo valore reale, un valore estetico, spettacolare. Il carattere commerciale delle esposizioni è ancora evidente nelle medaglie che decorano le etichette di alcune bevande alcoliche e di alcuni manufatti, erano importanti competizioni tra produttori, 
con premi attribuiti da una giuria spesso internazionale che davano lustro al marchio e ne garantivano la qualità.

5 Attraverso materiali e tecniche diverse i prodotti diventano la meta del pellegrinaggio di massa che vuole vedere ed interagire con "il nuovo", come si vede nel testo di Francesca Misiano.

6 Il prodotto è usato per rappresentare il risultato di uno sviluppo collettivo e, spesso, giustificarne le ambizioni dimostrando il grado di benessere raggiunto assimilandolo a quello di civilizzazione.

7 Con i prodotti, fossero seriali o artigianali, si costruivano gerarchie basate sulla condizione di "superiorità-inferiorità" tra le nazioni attraverso l'oggettivazione e la quantificazione dei risultati ottenuti, in una competizione globale che doveva indicare all'umanità il cammino corretto per la realizzazione del progresso. La ricerca di una collocazione adeguata nella "gerarchia delle nazioni" aumentò nell'epoca dei totalitarismi e della guerra fredda quando, accanto alla borghesia capitalista e agli Stati democratici con i loro messaggi di superiorità nazionale, trovò spazio anche l'esibizione attraverso la spettacolarizzazione dei prodotti delle industrie di Stato, delle innovazioni tecnologiche e delle opere pubbliche realizzate con la guida del "partito" e del leader.

Un'automobile, una riproduzione in scala di un'infrastruttura o una puleggia si convertivano in ingranaggi della macchina per il consenso internazionale che identificava l'ideologia con la merce.

9 Benjamin scriveva che «Le esposizioni mondiali trasfigurano il valore di scambio delle merci: creano un ambito in cui il loro valore d'uso passa in secondo piano: inaugurano una fantasmagoria in cui l'uomo entra per lasciarsi distrarre» ${ }^{5}$.

Occorre solo un biglietto d'ingresso per essere investiti da fantasmagorie e distrarsi osservando "il prodotto" esposto in un contesto spettacolare, celebrando un rituale sociale che elimina le differenze di classe per configurare un pubblico di massa, modellandone gusti e preferenze.

11 Le tecniche per la "messa in scena" non sono molto differenti da quelle che applicavano, e applicano, $i$ vetrinisti in forze agli esercizi commerciali. Non è un caso che i più noti architetti, ingegneri, artisti, designer industriali, pubblicitari e propagandisti annoverino almeno una partecipazione a questa gara giocata a colpi di fantasmagorie.

12 Lo svago che si offre in un'esposizione è diverso rispetto a quello tradizionale, come sostiene Benjamin: «L'industria del divertimento raffina e accresce la varietà dei comportamenti reattivi delle masse. Le prepara così per il successivo trattamento attraverso la pubblicità. È dunque ben fondato il suo legame con le esposizioni universali» ${ }^{6}$.

13 Il principe Giuseppe Carlo Napoleone di Francia nel 1857, presentò all'Imperatore Napoleone III, il Rapport sur l'exposition universelle de $1855^{7}$ dove si formulava una teoria del pubblico, considerato «le premier et le principal juge d'une Exposition». Il principe affermava che il pubblico di un'esposizione si costruisce socialmente, in maniera completa e collaborativa con il resto del sistema, solo quando è educato per farlo. La conclusione alla quale arriva è che un'esposizione si converte in uno spettacolo che fa coincidere il desiderio capitalista di una clientela per i suoi prodotti con quello delle 
distinte classi sociali, in maggioranza metropolitane, interessate tanto all'educazione, quanto al divertimento offerto da una esposizione industriale.

Potremmo definire gli organizzatori delle prime esposizione come "tecnici del divertimento", che gettavano le basi per la comprensione delle esigenze anche psicologiche delle masse, delle caratteristiche del gusto collettivo e che, attraverso queste informazioni, riuscivano a trasformare il pubblico di un' esposizione in una realtà fisica e onnipresente che si trasformava con il passo del tempo, adeguandosi ai cambiamenti sociali e politici, modificando i propri gusti nella stessa forma in cui cambiava la moda; nello stesso tempo, lo sviluppo tecnologico generava per ogni edizione nuove fantasmagorie e forme di divertimento per le grandi masse urbane. Neanche lo sport, come osserva Simón è esente da questa funzione, si celebrano tornei e gli spazi espositivi si concepiscono anche come luoghi per l'attività sportiva agonistica e amatoriale.

Mega-eventi ${ }^{8}$ che modificano e ingrandiscono gli spazi urbani accompagnando i mutamenti politici, culturali ed economici. Celebrano il presente, glorificano il passato e prefigurano ottimistici futuri. Come scrive Pellegrino nel suo saggio dedicato alle edizioni del XIX secolo, «interessavano all'epoca quantità enormi di persone, fino al culmine di 50 milioni di ingressi all'esposizione parigina del 1900, e raggiungevano un pubblico estremamente vasto; il loro effetto era però amplificato dal fatto che su di esse si innestava una vastissima produzione di rappresentazioni, figurative e scritte.» I messaggi propagandistici e pubblicitari sono veicolati verso l'esterno del recinto espositivo, canale e spazio di ricezione, aggiungendo un pubblico più ampio, che giustifica i costi sostenuti dagli espositori. Reportages, cartoline postali, libri, film ambientati tra i padiglioni ed altri prodotti culturali canalizzano l'esposizione verso il pubblico internazionale, stabilendo una gerarchia e una censura dei contenuti offerti nei padiglioni e partecipando in questa funzione alla costruzione degli immaginari collettivi9.

16 Come scritto da Coglitore in conclusione del saggio che apre quest'antologia, nelle ultime decadi l'Esposizione sembra ormai sciolta «in un insieme di riferimenti simbolici che aveva già oltrepassato se stesso, e dal moderno al post-moderno - all'interno di società segnate dall'avidità dei mercati finanziari, dall'aggressività dei messaggi pubblicitari, dall'invadenza della televisione, da flussi ininterrotti di informazioni che scorrevano adesso attraverso reti telematiche».

17 Prima di questa "decadenza", le esposizioni avevano raggiunto in un breve lasso di tempo dalla prima edizione un tal successo che nel 1928 a Parigi venne istituito il Bureau International des Expositions ${ }^{10}$ (B.I.E.), con lo scopo di classificare ufficialmente le differenti esposizioni che si organizzavano in tutto il mondo, con lo scopo di mettere un freno al imperante caos di denominazioni che rischiava di svalutare "il marchio" di cui facevano gala le principali capitali economiche occidentali. Il primo protocollo firmato dai paesi membri nel $1928^{11}$, entrato in vigore nel 1931, faceva una chiara differenziazione ${ }^{12}$ tra quelle di tipo generale universale, che duravano dodici mesi e dove i paesi espositori costruivano i propri padiglioni, e le esposizioni specializzate internazionali, che duravano sei mesi, e dove la realizzazione dei padiglioni era responsabilità dell'organizzazione del Paese ospitante. A queste si accompagnavano le fiere di settore e quelle nazionali e imperiali, dove si rappresentavano le colonie. per il pubblico metropolitano. 
Il colonialismo, inteso come processo non soltanto politico ed economico, ma anche culturale, trovò nel momento espositivo uno degli strumenti più efficaci di diffusione $\mathrm{e}$ di fissazione dell'alterità nell'immaginario europeo tardo ottocentesco.

A partire dalla prima edizione di Londra, nel 1851, le esposizioni universali, con la loro caratteristica organizzazione secondo padiglioni nazionali, ebbero lo scopo di affermare e mostrare il carattere nazionale di ogni paese partecipante. Il mondo coloniale irruppe in questo schema come un fenomeno che doveva essere rappresentato in maniera vicina e distante allo stesso tempo.

Se le conquiste oltremare dovevano essere 'nazionalizzate' ed esposte all'interno dei padiglioni nazionali per sottolineare la grandezza del paese conquistatore, i paesi e le popolazioni colonizzati dovevano essere rappresentati distanti, esotici e diversi dal colonizzatore.

21 Le esposizioni, come osserva Cini per il caso corso, «consentono anche di riflettere su alcuni aspetti inerenti la dialettica fra Stato nazionale e singole aree regionali rispetto alle molteplici problematiche che innervarono tale relazione nel corso del XIX secolo, fra cui quella dell'integrazione economica fra centro e periferie».

Il colonialismo, inteso come processo non soltanto politico ed economico, ma anche culturale, trovò nel momento espositivo uno degli strumenti più efficaci di diffusione $\mathrm{e}$ di fissazione dell'alterità nell'immaginario europeo tardo ottocentesco.

Tanto nella dimensione nazionale come in quella universale, le esposizioni diventano templi e monumenti delle religioni civili e ne inglobano la simbologia, come descritto nel saggio di Cimadomo e Lecardane e in quello di Vilanova i Vila-Abadal che affrontano il legame tra esposizioni, architettura ed ideologia inserendosi nel dibattito sulle relazioni tra arte e potere ${ }^{13}$.

\section{L'esposizione della propaganda}

Le esposizioni rappresentano un fenomeno strettamente connesso con la modernità e con la contemporaneità la cui analisi può originare o integrarsi in riflessioni più ampie sulla società e la geopolitica sono cataloghi di identità e di argomentazioni tese a dimostrare le caratteristiche di superiorità nazionale o culturale.

Gli stessi contemporanei percepivano l'organizzazione e le intenzioni che soggiacevano alla realizzazione di questi simulacri identitari in cui Walter Benjamin rintracciava «l'occulto schema costruttivo dei musei». Già Nel 1891, il filosofo Plechanov ${ }^{14}$ considerava le esposizioni come uno strumento per convincere della necessità, superiorità e convenienza di un determinato modello sociale o di sviluppo, avendo osservato nelle modalità con cui la borghesia francese aveva organizzato l'evento per il centenario della rivoluzione per dimostrare al proletariato la possibilità e la necessità di una trasformazione sociale dimostrata dallo sviluppo dei mezzi di produzione raggiunto nelle nazioni "civilizzate" che superava le «osate fantasie dei pensatori utopici del secolo passato».

In strutture destinate alla demolizione o alla riconversione con una narrazione fatta di immagini, fantasmagorie e rappresentazioni grafiche di dati si sono costruiti immaginari collettivi. Nei recinti espositivi si manifesta una continua mitopoiesi nella diffusione di narrazioni univoche che, spesso accennando alle radici, descrivono il presente e provano a persuadere i visitatori con visioni di futuro. 
Il passato è usato per celebrare le origini, giustificare il presente e motivare la visione ottimistica del futuro. I reperti archeologici, come scrive Noel «souvent support aux idéologies contemporaines chez les commentateurs et le grand public» e accompagnano le rivendicazioni, siano esse sociali, nazionali o imperiali. Le nazioni che vogliono raccontare la propria "missione storica" o rivendicare diritti e colonie cercano nel passato gli argomenti per persuadere l'opinione pubblica, come per le colonne romane trovate nel deserto etiopico esposte dagli italiani nel 1937 a Parigi.

I governi nelle esposizioni a volte riscrivono o censurano la storia nazionale, come scrive Gonçalves riguardo il caso portoghese: «La nécessité pour le gouvernement de se donner une légitimité passait nécessairement par la diffusion d'une image cohérente et unifiée du Portugal et par celle d'une nation contre laquelle rien ne résistait, toujours digne et victorieuse car placée sous la protection de la providence».

Durante la Guerra Fredda e l'epoca totalitaria le eredità del passato e le immagini del presente servono a prefigurare e rendere accettabile un futuro in cui l'altro non esiste perché è stato sconfitto. I contenuti dei padiglioni delle nazioni nemiche diventano argomentazioni da usare nella propaganda del nemico, per costruire l'odio e favorire il conflitto. Come descritto nel testo di Cantano e Zapico si realizzano "esposizioni anti-“", cataloghi di argomentazioni a favore dell'odio e del disprezzo che si inseriscono perfettamente nei meccanismi propagandistici autoritari.

\section{Le colonie in esposizione}

Anche se Edward Said ha concentrato il suo lavoro su come la letteratura abbia contribuito alla formazione dell'idea di impero nella società britannica, e a promuovere un consenso all'espansione, la più ampia e generale connessione tra cultura e imperialismo che lo studioso palestinese è riuscito a evidenziare in maniera così limpida e convincente non può non essere presa in considerazione anche nel quadro dell'analisi della rilevanza delle esposizioni nella formazione di una coscienza coloniale e imperiale in Europa ${ }^{15}$.

31 Come ha messo in evidenza Michael Vann per quanto concerne il colonialismo francese, le esposizioni coloniali ci consentono, seguendo un filo cronologico, di individuare come i cambiamenti politici sui metodi e modelli di amministrazione oltremare venissero rielaborati e riportati negli spazi espositivi ${ }^{16}$. Sulle esposizioni inerenti il colonialismo francese si sofferma in questo numero di Diacronie Christelle Lozère Bernard, che analizza le esposizioni provinciali e il loro ruolo nel raccontare l'oltremare francese nelle regioni periferiche della nazione, da Marsiglia a Bordeaux, da Cherbourg a Beauvais. Nel suo lavoro Lozère-Bernard mette inoltre in evidenza come nelle prime esposizioni provinciali della seconda metà dell'Ottocento si riproduca uno degli assi portanti dell'ideologia coloniale europea; la conquista coloniale viene irrimediabilmente associata al progresso europeo, rendendo anche negli spazi espositivi quella convinzione ideologica che giustificava la conquista coloniale rappresentandola come obbligo di una civiltà progredita nei confronti delle altre.

Le selezioni operate dai diversi paesi per allestire le esposizioni coloniali, e per organizzare gli spazi dedicati alle colonie all'interno di quelle universali, costituirono uno dei passaggi chiave di quel processo eurocentrico di rappresentazione dell'altro che Said ha definito orientalismo. Su questo aspetto riflette in questo numero Nader 
Meddeb, che concentra il suo saggio sui padiglioni dedicati alla Tunisia e all'architettura degli spazi espositivi, estrema sintesi, ma anche evidente prova, di un orientalismo che rivela l'ibridità prodotta dalla conquista coloniale. Destinate a un pubblico più ampio rispetto a quello al quale erano destinate le pubblicazioni scientifiche e la letteratura, gli spazi espositivi contribuirono in maniera concreta a definire i connotati dell'altro, a farne circolare una 'certa' rappresentazione, e a ufficializzare e istituzionalizzare quelle caratteristiche come 'date'. La concretezza delle rappresentazioni espositive portava in casa dei colonizzatori il mondo coloniale, la cui conoscenza doveva necessariamente essere mediata vista l'impossibilità che le popolazioni colonizzatrici potessero avere testimonianza diretta del mondo coloniale.

Per poter informare e istruire il cittadino europeo su quel mondo che le nazioni europee, e perciò egli stesso, andavano a conquistare, la conoscenza dell'oltremare, delle popolazioni assoggettate, degli usi e dei costumi, dei prodotti e delle consuetudini doveva necessariamente divenire oggetto di racconto. Gli allestimenti espositivi affiancarono in questo senso la letteratura, fornendo un racconto visivo e tangibile dell'esperienza coloniale. La ricostruzione delle abitazioni degli indigeni, l'esposizione di manufatti e l'allestimento di grandi mappe, orientavano il visitatore all'interno del mondo coloniale, trasformandolo in parte da spettatore a protagonista. nel fornire ai visitatori una conoscenza diretta, di un mondo del quale la maggior parte di essi non avrebbe potuto avere esperienza diretta e concreta. L'analisi delle modalità con le quali queste esposizioni raccontarono l'esperienza rappresenta un importante momento di comprensione dell'intreccio tra politica, economia e cultura nella dimensione coloniale, e, al contempo, ci offre la possibilità di individuare alcuni aspetti di quell'esperienza che, nonostante la fine del colonialismo, hanno continuato a influenzare la cultura europea durante il XX secolo.

\section{NOTE}

1. RYDELL, Robert W., GWINN, Nancy E., Fair Representations: World's Fairs and the Modern World, Amsterdam, VU University Press, 1994.

2. BASSIGNANA, Pier Luigi, Le feste popolari del capitalismo: esposizioni d'industria e coscienza nazionale in Europa, 1798-1911, Torino, Umberto Allemandi, 1997.

3. HOBSBAWM, Eric J., Il trionfo della borghesia, Roma-Bari, Laterza, 1994.

4. CLASEN, Wolfgang, Expositions, Exhibits, Industrial and Trade Fairs, New York, Praeger, 1968.

5. BENJAMIN, Walter, L'opera d'arte nell'epoca della sua riproducibilitá tecnica, Torino, Einaudi, 1995, p. 151.

6. BENJAMIN, Walter, I «passages» di Parigi, Torino, Einaudi, 2000, p. 211.

7. BONAPARTE, Napoléon J.P.C., Rapport sur l'exposition universelle de 1855 présenté á l'Empereur par S.A.I. le Prince Napoléon, président de la commission, París, Imprimerie impériale, 1856.

8. ROCHE, Maurice, Mega-Events and Modernity: Olympics and Expos in the Growth of Global Culture, London, Routledge, 2000.

9. CASTORIADIS, Cornelius, L'institution imaginaire de la société, Paris, Éditions du Seuil, 1975. 
10. GALOPIN, Marcel, Les Expositions internationales au XXe siécle et le Bureau International des Expositions, París, L'Harmattan 1997.

11. International Exhibitions Bureau, 75th anniversary of he Convention of the International Exhibitions Bureau signed at París on 22 November 1928 relating [to] international exhibitions, Paris, Bureau International des Expositions, 2004.

12. WATERS, Honeric William, History of Fairs and Expositions: Their Classification, Functions and Values, London, Reis Bros., 1939.

13. ADES, Dawn (ed.), Art and Power: Europe Under the Dictators 1930-45, London, Thames and Hudson, 1995.

14. PLECHANOW, Georgi Walentinowitsch, «Wie die bourgeoise ihrer Revolution gedenkt», in Die Neue Zeit, IX, 1/1891, p. 138.

15. SAID, Edward, Cultura e imperialismo, Roma, Gamberetti Editrice, 1988.

16. Cfr. VANN, Michael G., 'All the World's a Stage', Especially in the Colonies: L'Exposition de Hanoi, 1902-3, in EVANS, Martin (ed.), Empire and Culture. The French Experience, 1830-1940, New York, Palgrave macmillan, 2004, pp. 181-191, p. 181.

\section{AUTORI}

\section{FRANCESCOMARIA EVANGELISTI}

$\mathrm{PhD}$ in "Comunicazione e critica della cultura" presso l'Università di Siviglia, è ricercatore in "Storia della propaganda ed analisi della comunicazione politica" per COMPOLITICAS. Attualmente dirige la comunicazione del Movimento Cinque Stelle Lazio.

\section{ALESSANDRO PES}

Ricercatore in Storia Contemporanea presso il Dipartimento di Scienze Sociali e delle Istituzioni dell'Università di Cagliari, si occupa di storia del fascismo e del colonialismo italiano. Su questi temi ha pubblicato: La costruzione dell'impero fascista. Politiche di regime per una società coloniale (Roma, Aracne Editrice, 2010); Bonificare gli italiani. La Società Bonifiche Sarde tra risanamento e colonizzazione nell'Italia fascista (Cagliari, Am\&D Edizioni, 2013). 